TRANSACTIONS OF THE

AMERICAN MATHEMATICAL SOCIETY

Volume 355, Number 1, Pages 177-195

S 0002-9947(02)03118-5

Article electronically published on September 6, 2002

\title{
INTEGRATION OF MULTIVALUED OPERATORS AND CYCLIC SUBMONOTONICITY
}

\author{
ARIS DANIILIDIS, PANDO GEORGIEV, AND JEAN-PAUL PENOT
}

\begin{abstract}
We introduce a notion of cyclic submonotonicity for multivalued operators from a Banach space $X$ to its dual. We show that if the Clarke subdifferential of a locally Lipschitz function is strictly submonotone on an open subset $U$ of $X$, then it is also maximal cyclically submonotone on $U$, and, conversely, that every maximal cyclically submonotone operator on $U$ is the Clarke subdifferential of a locally Lipschitz function, which is unique up to a constant if $U$ is connected. In finite dimensions these functions are exactly the lower $\mathrm{C}^{1}$ functions considered by Spingarn and Rockafellar.
\end{abstract}

\section{INTRODUCTION}

We deal with the integration of a multivalued operator considered as the inverse process of taking the subdifferential of a function. This important question has been tackled by several authors: see for instance [20] (for the Fenchel-Moreau subdifferential of a convex function), [12, [19], [17] (for the Clarke subdifferential in finite dimensions), [4], [26] (for the Clarke subdifferential in infinite dimensions), [1] (for the moderate subdifferential of Michel-Penot in finite dimensions) and [25], 27] (for various subdifferentials of a lower semicontinuous function). The first mentioned result concerns the case of monotone operators: in [20] Rockafellar shows that any cyclically monotone operator $T$ is included in the subdifferential of a lower semicontinuous convex function, with equality if $T$ is maximal cyclically monotone. Janin 12 introduces a concept of cyclic submonotonicity in finite-dimensional Euclidean spaces and uses it to integrate (in the preceding sense) locally bounded operators satisfying that condition into locally Lipschitz functions. In a different context, a concept of cyclicity has also been used by Qi in [19, where the author characterizes operators that coincide with a subdifferential of some locally Lipschitz function, using the Lebesgue measure and (implicitly) the Rademacher theorem. Elaborating upon these ideas, Borwein and Moors 3 introduce and study the class $S_{e}(X)$ of essentially smooth (locally Lipschitz) functions, that is, functions $f$ whose Clarke subdifferential $\partial^{C} f$ is single-valued in the complement of a Haar null set. One of the main features of this class stems from the fact that for every $f \in S_{e}(X)$, the problem of finding a locally Lipschitz function $g$ such that $\partial^{C} g \subseteq \partial^{C} f$ has a unique

Received by the editors May 4, 2000.

2000 Mathematics Subject Classification. Primary 49J52, 47H05; Secondary 58C20.

Key words and phrases. Integration, subdifferential, submonotone operator, subsmooth function.

The research of the first author was supported by the TMR grant ERBFMBI CT 983381.

A major part of this work was accomplished while the second author was visiting the University of Pau under the NATO grant CB/JB SC105 N0 44/96165. 
solution modulo a constant (i.e., $g=f+c$ ). In [4, Borwein, Moors and Shao extend the results of Qi [19] to separable Banach spaces, using line integrals and Christensen's generalization of the Rademacher theorem via Haar null sets. Their result is further improved by Wang [26, who characterizes the class of integrable locally bounded operators in separable Banach spaces. These operators are called in $[19$ and [26] "cyclically normal".

In another line of research, Spingarn [24] showed that in finite dimensions, lower $C^{1}$ functions (i.e., functions arising as maxima of compactly indexed families of $C^{1}$ functions) are characterized by the fact that their Clarke subdifferentials are strictly submonotone operators with nonempty values. This last notion was extended to infinite dimensions by Georgiev in [10], 11] (see the definition of directional strict submonotonicity in Section 2). Functions with such subdifferentials (herein called subsmooth) are always regular (in the sense of Clarke [6]) and semi-smooth (in the sense of Mifflin [14]), see [24] and [11. In particular, subsmooth functions have "small" (namely minimal $w^{*}$-cusco) Clarke subdifferentials. Let us recall that, in general, Lipschitz functions have "generically" very large Clarke subdifferentials ([26]). In finite dimensions, as the notions of strict submonotonicity and directional strict submonotonicity coincide [11, a function $f$ is subsmooth if, and only if, it is lower $C^{1}$.

Our main results rely on a notion of cyclic submonotonicity introduced here; in finite dimensions it coincides with the definition of Janin [12]. Using this concept, we show that if $U$ is an open subset of a Banach space $X$, then

- the subdifferential of every subsmooth function (defined on $U$ ) is maximal cyclically submonotone (on $U$ );

- if $U$ is connected and $f, g$ are subsmooth functions on $U$ such that $\partial^{C} f=$ $\partial^{C} g$, then $f=g+c$ for some constant $c \in \mathbb{R}$; and

- every maximal cyclically submonotone operator on $U$ is the subdifferential of a subsmooth function defined on $U$ (unique up to a constant if $U$ is connected).

A specific feature of our approach is that it does not depend on results from measure theory and is valid beyond the class of separable spaces.

Notation. Let us now fix our notation. We denote by $(X,\|\cdot\|)$ a Banach space, by $S_{X}$ its unit sphere and by $\left(X^{*},\|\cdot\|\right)$ its dual space. We also denote by $B_{r}(x)$ (resp. $B_{r}[x]$ ) the open (resp. closed) ball with center $x$ and radius $r$, and by $B_{r}^{*}(x)$ (resp. $B_{r}^{*}[x]$ ) the same objects in $X^{*}$. Let $2^{X^{*}}$ be the set of all subsets of $X^{*}$, and $\mathbb{R}$ (resp. $\mathbb{N}$ ) be the set of all real (resp. nonnegative integer) numbers. For any $k \in \mathbb{N}$, we set $\mathbb{N}_{k}:=\{1,2, \ldots, k\}$. For any $x, y$ in $X$, we denote by $[x, y]:=\left\{x_{t}=t x+(1-t) y\right.$, $0 \leq t \leq 1\}$ the closed segment with endpoints $x, y$. For any subset $K$ of $X$ and any $\delta>0$, we consider the $\delta$ - (open) neighborhood $B_{\delta}(K)$ of $K$ defined by $B_{\delta}(K):=$ $\{x \in X: \exists y \in K,\|x-y\|<\delta\}$. We also set $\operatorname{diam}(K):=\sup \{\|x-y\|: x, y \in K\}$ for the diameter of the set $K$. We denote by cone $(K)$ the cone generated by $K$, i.e., cone $(K):=\{\lambda x: \lambda \geq 0, x \in K\}$. For any subset $A$ of $X^{*}$, we denote by $\overline{c o} w^{w^{*}}(A)$ the $w^{*}$-closed convex hull of $A$. Finally, throughout the paper we shall assume that all functions $f$ are locally Lipschitz and we shall denote by $\operatorname{dom}(f)$ their domain. 


\section{Preliminaries}

Given a multivalued operator $T: X \rightrightarrows X^{*}$, we denote by $\operatorname{dom}(T)$ the set $\{x \in$ $X: T(x) \neq \emptyset\}$ and by $\operatorname{Gr}(T):=\left\{\left(x, x^{*}\right) \in X \times X^{*}: x^{*} \in T(x)\right\}$ (or simply $T$ if no confusion may arise) the graph of $T$. We also define the operators $\overline{c o}^{w^{*}}(T)$ by

$$
\overline{c o}^{w^{*}}(T)(x):=\overline{c o}^{w^{*}}(T(x))
$$

for all $x \in X$ and $\bar{T}: X \rightarrow 2^{X^{*}}$ by

$$
x^{*} \in \bar{T}(x) \Longleftrightarrow\left\{\begin{array}{c}
\exists\left\{x_{i}\right\}_{i} \text { in } X, \exists\left\{x_{i}^{*}\right\}_{i} \text { in } X^{*}: x_{i}^{*} \in T\left(x_{i}\right) \\
x=\lim _{i} x_{i}, \quad x^{*}=w^{*}-\lim _{i} x_{i}^{*}
\end{array}\right.
$$

where $\left\{x_{i}\right\}_{i}$ and $\left\{x_{i}^{*}\right\}_{i}$ denote, respectively, nets in $X$ and in $X^{*}$. Note that $\operatorname{Gr}(\bar{T})=$ $\overline{\operatorname{Gr}(T)}\|\cdot\| \times w^{*}$.

The operator $T: X \rightrightarrows X^{*}$ is said to be locally bounded at $x \in X$ if there exist $M>0$ and a neighborhood $B_{r}(x)$ of $x$ such that $u \in B_{r}(x)$ and $u^{*} \in T(u)$ imply $\left\|u^{*}\right\|<M$. Then $T$ is called locally bounded on a subset $U$ of $X$ if $T$ is locally bounded at all $x \in U$. (Note that $U$ is not necessarily a subset of $\operatorname{dom}(T)$.)

Furthermore, the operator $T$ is said to be $w^{*}$-upper-semicontinuous at $x \in X$ if for every $w^{*}$-open set $W \supset T(x)$, there exists an open ball $B_{r}(x)$ of $x$ such that $T(u) \subseteq W$ for every $u \in B_{r}(x)$. Let us note that if $T$ is locally bounded on $U$, then $\bar{T}$ (given by relation (2)) is $w^{*}$-upper-semicontinuous at every $x \in \operatorname{dom}(\bar{T}) \cap U$.

$w^{*}$-cusco mappings. A multivalued mapping $T: X \rightrightarrows X^{*}$ is said to be $w^{*}$-cusco on $U$ (2], [5], e.g.), if it is $w^{*}$-upper semicontinuous with nonempty $w^{*}$ compact convex values on $U$. A $w^{*}$-cusco mapping on $U$ that does not strictly contain any other $w^{*}$-cusco mapping with domain in $U$ is called minimal $w^{*}$-cusco on $U$.

Given an operator $S$, we can consider $w^{*}$-cusco mappings $T$ that are minimal under the property of containing $S$. In the important case of the following proposition, one can give a complete description of the minimal (in fact least) element of the family of $w^{*}$-cusco mappings containing $S$.

Proposition 1. Let $S$ be a densely defined locally bounded operator on an open subset $U$ of $X$ with values in $X^{*}$. Then the family of $w^{*}$-cusco mappings containing $S$ has a least element $T$ given by the formula

$$
T(x)=\overline{c o}^{w^{*}} \bigcap_{\varepsilon>0} \overline{\left\{S\left(x^{\prime}\right): x^{\prime} \in B_{\varepsilon}(x) \cap \operatorname{dom}(S)\right\}}{ }^{w^{*}} .
$$

Proof. In [2, Proposition 1.3] (see also [15, Proposition 1.2]), the following formula for the operator $T$ is given:

$$
T(x)=\bigcap_{\varepsilon>0} \overline{c o}^{w^{*}}\left\{S\left(x^{\prime}\right): x^{\prime} \in B_{\varepsilon}(x) \cap \operatorname{dom}(S)\right\} .
$$

In order to justify (3), let us set

$$
R(x):=\bigcap_{\varepsilon>0} \overline{\left\{S\left(x^{\prime}\right): x^{\prime} \in B_{\varepsilon}(x) \cap \operatorname{dom}(S)\right\}}{ }^{*} .
$$

Since $S$ is locally bounded on $U$, it is easily seen that $R(x)=\bar{S}(x)$ (given by relation (2) ) and that $R$ is the smallest $w^{*}$-upper-semicontinuous multivalued mapping containing $S$. Thus $R(x) \subseteq T(x)$ and $\overline{c o} w^{*}(R(x)) \subseteq T(x)$ for each $x \in U$. 
Since $\overline{c o}^{w^{*}}(R(x))$ is $w^{*}$-cusco (see [5, Proposition 2.7], e.g.) and $T$ is the minimal $w^{*}$-cusco containing $S$, we get $\overline{c o}^{w^{*}}(R)=T$.

The preceding proposition has an interesting (and immediate) consequence on the representation of the Clarke subdifferential in certain Banach spaces. We recall that the Clarke generalized derivative of a locally Lipschitz function $f$ at a point $x \in \operatorname{dom}(f)$ is defined for all $u \in X$ as follows:

$$
f^{o}(x ; u)=\limsup _{(y, t) \rightarrow\left(x, 0^{+}\right)} \frac{f(y+t u)-f(y)}{t},
$$

and the Clarke subdifferential of $f$ at $x \in \operatorname{dom}(f)$ by

$$
\partial^{C} f(x)=\left\{x^{*} \in X^{*}:\left\langle x^{*}, u\right\rangle \leq f^{o}(x, u), \forall u \in X\right\} .
$$

For all $x \in \operatorname{dom}(f)$, we have $\partial^{C} f(x) \neq \emptyset$.

Let us also recall the definitions of other usual subdifferentials that will occur in the sequel:

- the Fréchet subdifferential $\partial^{F} f(x)$

$$
\partial^{F} f(x):=\left\{x^{*} \in X^{*}: f(y) \geq f(x)+\left\langle x^{*}, y-x\right\rangle+o(y-x), \forall y \in X\right\},
$$

where $o: X \rightarrow R$ is some real-valued function satisfying $\lim _{u \rightarrow 0} \frac{o(u)}{\|u\|}=0$;

- the Hadamard subdifferential $\partial^{H} f(x)$

$$
\partial^{H} f(x)=\left\{x^{*} \in X^{*}:\left\langle x^{*}, u\right\rangle \leq \liminf _{(w, t) \rightarrow\left(u, 0^{+}\right)} \frac{f(x+t w)-f(x)}{t}, \forall u \in X\right\} .
$$

Let us note that if $f$ is locally Lipschitz, then for all $u \in X$,

$$
d f(x, u):=\liminf _{(w, t) \rightarrow\left(u, 0^{+}\right)} \frac{f(x+t w)-f(x)}{t}=\liminf _{t \searrow 0^{+}} \frac{f(x+t u)-f(x)}{t}:=f^{\prime}(x, u),
$$

so that the Hadamard derivative coincides with the Gâteaux derivative of $f$ when they exist.

Let us now recall that in every Asplund space, the Clarke subdifferential $\partial^{C} f$ of a locally Lipschitz function $f$ is given by the following formula of Preiss ( $\llbracket 18$. Remark 2.3]):

$$
\partial^{C} f(x)=\bigcap_{\varepsilon>0} \overline{c o}^{w^{*}}\left\{D^{F} f\left(x^{\prime}\right): x^{\prime} \in B_{\varepsilon}(x) \cap \operatorname{dom}\left(D^{F} f\right)\right\},
$$

while, if $X$ has a Gâteaux smooth renorming,

$$
\partial^{C} f(x)=\bigcap_{\varepsilon>0} \overline{c o}^{w^{*}}\left\{D^{H} f\left(x^{\prime}\right): x^{\prime} \in B_{\varepsilon}(x) \cap \operatorname{dom}\left(D^{H} f\right)\right\},
$$

where $D^{F} f(x)$ (resp. $D^{H} f(x)$ ) denotes the Fréchet (resp. Hadamard) derivative of $f$ at $x$ and $\operatorname{dom}\left(D^{F} f\right)$ (resp. $\left.\operatorname{dom}\left(D^{H} f\right)\right)$ is the domain of $D^{F} f$ (resp. $\left.D^{H} f\right)$.

Since $\partial^{C} f$ is a $w^{*}$-cusco mapping ([3]), combining Proposition 1 with formulas (6) and (7), we obtain in view of [18, Theorem 2.4] the following corollary.

Corollary 2. For every (locally Lipschitz) function $f$ on $X$ we have:

(i) if $X$ is an Asplund space, then

$$
\partial^{C} f(x)=\overline{c o}^{w^{*}} \bigcap_{\varepsilon>0} \overline{\left\{D^{F} f\left(x^{\prime}\right): x^{\prime} \in B_{\varepsilon}(x) \cap \operatorname{dom}\left(D^{F} f\right)\right\}} w^{*}
$$


(ii) if $X$ has an equivalent Gâteaux differentiable norm, then

$$
\partial^{C} f(x)=\overline{c o}^{w^{*}} \bigcap_{\varepsilon>0} \overline{\left\{D^{H} f\left(x^{\prime}\right): x^{\prime} \in B_{\varepsilon}(x) \cap \operatorname{dom}\left(D^{H} f\right)\right\}}{ }^{*} .
$$

Submonotone and strictly submonotone mappings. In 1981, J. Spingarn 24] introduced the notion of a strictly submonotone mapping in a finite-dimensional space. His definition is naturally extended to infinite dimensions as follows: a multivalued mapping $T: X \rightrightarrows X^{*}$ is said to be strictly submonotone (for short, $s$ submonotone) at $x \in X$ provided that for any $\varepsilon>0$, there exists $\delta>0$ such that

$$
\frac{\left\langle x_{1}^{*}-x_{2}^{*}, x_{1}-x_{2}\right\rangle}{\left\|x_{1}-x_{2}\right\|} \geq-\varepsilon
$$

whenever $x_{i} \in B_{\delta}(x), x_{i}^{*} \in T\left(x_{i}\right), i=1,2$, and $x_{1} \neq x_{2}$.

The operator $T: X \rightrightarrows X$ is called submonotone at $x$, if (10) holds under the additional assumption $x_{2}=x$. (Note that $T$ is submonotone at every $x \notin \operatorname{dom}(T)$ and s-submonotone at every $x \notin \overline{\operatorname{dom}}(T)$.)

Appropriate directional versions of these notions have been introduced in [10] (see also [1] and [16]): an operator $T: X \rightrightarrows X^{*}$ is called directionally strictly submonoton 11 (for short, $d s$-submonotone) at $x$, if for every $e \in S_{X}$ and $\varepsilon>0$, there exists $\delta>0$ such that

$$
\frac{\left\langle x_{1}^{*}-x_{2}^{*}, x_{1}-x_{2}\right\rangle}{\left\|x_{1}-x_{2}\right\|} \geq-\varepsilon
$$

whenever $x_{i} \in B_{\delta}(x), x_{i}^{*} \in T\left(x_{i}\right), i=1,2, x_{1} \neq x_{2}$ and $\left\|\frac{x_{1}-x_{2}}{\left\|x_{1}-x_{2}\right\|}-e\right\|<\delta$.

The operator $T: X \rightrightarrows X^{*}$ is called directionally submonotone (for short, $d$ submonotone) at $x$, if (11) holds under the additional assumption $x_{2}=x$.

It is easily seen that if (11) holds uniformly for all directions $e \in S_{X}$, then $T$ is s-submonotone at $x$. Similarly, if (11) holds uniformly for $x_{2}=x$, then $T$ is submonotone at $x$. If $X=\mathbb{R}^{n}$, the compactness of the unit sphere in $\mathbb{R}^{n}$ entails that an operator $T$ is ds-submonotone (resp. d-submonotone) if, and only if, it is s-submonotone (resp. submonotone).

Given a nonempty subset $U$ of $X$, we say that $T$ is s-submonotone (resp. submonotone, ds-submonotone, d-submonotone) on $U$, if $T$ has the corresponding property at every $x \in U$.

Let us recall from [11, Theorem 2.4] that every ds-submonotone operator $T$ on $X$ is locally bounded on $\operatorname{int} \operatorname{dom}(T)$. The definition of ds-submonotonicity (relation (11) is reminiscent of monotonicity and can be considered as a limiting variant of it. It can also be considered as a mild continuity condition, since any continuous function $g: U \rightarrow X^{*}$ can be seen as a (single-valued) s-submonotone operator on $U$. Thus, every monotone operator is s-submonotone, while the converse is not true. The class of s-submonotone operators is stable under addition and is relatively large.

\section{A SUFFICIENT CONDITION FOR INTEGRATION}

In this section we give sufficient conditions for integrating multivalued operators. We first need some terminology. Given a segment $[x, y]$, a finite sequence $\left\{x_{i}\right\}_{i=1}^{k}$

\footnotetext{
1 "strictly submonotone" according to the terminology of [10, 11].
} 
of $[x, y]$ is called a subdivision of the segment $[x, y]$, if $x_{1}=x, x_{k}=y$ and

$$
\sum_{i=1}^{k-1}\left\|x_{i+1}-x_{i}\right\|=\|x-y\| .
$$

A polygonal path $\left[w_{h}\right]_{h=1}^{m}$ is a union of consecutive segments; it is said to be closed if $w_{m}=w_{1}$. A finite sequence $\left\{x_{i}\right\}_{i=1}^{n}$ is called a subdivision of the path $\left[w_{h}\right]_{h=1}^{m}$ if there exists an increasing sequence $1=k_{1}<k_{2}<\ldots<k_{m}=n$ such that for $1 \leq h \leq m-1,\left\{x_{i}\right\}_{i=k_{h}}^{k_{h+1}}$ is a subdivision of the segment $\left[w_{h}, w_{h+1}\right]$.

The following definition is a reformulation in infinite dimensions of a property introduced by Janin 12] for the class of bounded operators defined on compact subsets of $\mathbb{R}^{n}$.

Definition 3. An operator $T: X \rightrightarrows X^{*}$ is called radially cyclically submonotone on a subset $U$ of its domain if for any closed polygonal path $\left[w_{h}\right]_{h=1}^{m} \subseteq U$ and any $\varepsilon>0$, there exists $\delta>0$, such that for any subdivision $\left\{x_{i}\right\}_{i=1}^{n}$ of $\left[w_{h}\right]_{h=1}^{m}$ satisfying $\left\|x_{i+1}-x_{i}\right\|<\delta($ for $i=1,2, \ldots, n)$ and any $x_{i}^{*} \in T\left(x_{i}\right)$ one has

$$
\sum_{i=1}^{n-1}\left\langle x_{i}^{*}, x_{i+1}-x_{i}\right\rangle \leq \varepsilon .
$$
4.

The proof of the following result borrows ideas from [12, mainly in steps 1 and

Theorem 4. Let $T: X \rightrightarrows X^{*}$ be locally bounded and radially cyclically submonotone on an open subset $U$ of $\operatorname{dom}(T)$. Suppose that $T$ is $d$-submonotone, or more generally, that for any $x \in U, x^{*} \in T(x), u \in X$ one has

$$
\liminf _{t \rightarrow 0^{+}} \sup _{y^{*} \in T(x+t u)}\left\langle y^{*}-x^{*}, u\right\rangle \geq 0 .
$$

Then there exists a locally Lipschitz function $f: U \rightarrow \mathbb{R}$ such that $T \subseteq \partial^{H} f$ on $U$. If, in addition, $T$ is submonotone, then $T \subseteq \partial^{F} f$ on $U$.

Proof. Case 1: Let us first suppose that $U$ is connected. Then let $V$ be the set of $(x, y) \in U \times U$ such that $[x, y] \subseteq U$. Given $(x, y) \in V$ and $\sigma>0$, let us denote by $S_{\sigma}(x, y)$ the set of subdivisions $\left\{x_{i}\right\}_{i=1}^{n}$ of the segment $[x, y]$ such that $x_{1}:=x$, $x_{n}:=y$, and $\left\|x_{i+1}-x_{i}\right\|<\sigma$ for $i=1,2, \ldots, n-1$.

We consider the function $g(\cdot, \cdot): V \rightarrow \mathbb{R} \cup\{+\infty\}$ given by

$$
g(x, y):=\inf _{\sigma>0} \sup \left\{\sum_{i=1}^{n-1}\left\langle x_{i}^{*}, x_{i+1}-x_{i}\right\rangle:\left\{x_{i}\right\}_{i=1}^{n} \in S_{\sigma}(x, y), x_{i}^{*} \in T\left(x_{i}\right)\right\} .
$$

Since $T$ is locally bounded, a compactness argument shows that for any $(x, y) \in V$, there exist $k>0$ and $\rho>0$ such that for all $\left(x^{\prime}, y^{\prime}\right) \in B_{\rho}(x) \times B_{\rho}(y)$, we have $\left(x^{\prime}, y^{\prime}\right) \in V$ and

$$
\left|g\left(x^{\prime}, y^{\prime}\right)\right| \leq k\left\|x^{\prime}-y^{\prime}\right\| .
$$

Let us now fix some $x_{0}$ in $U$ and define $f: U \rightarrow \mathbb{R} \cup\{+\infty\}$ as follows:

$$
f(x):=\sup \left\{\sum_{h=1}^{m-1} g\left(w_{h}, w_{h+1}\right)\right\}
$$


where the supremum is taken over all $m \geq 2$ and all polygonal paths $\left[w_{h}\right]_{h=1}^{m}$ with $w_{1}:=x_{0}$ and $w_{m}:=x$ such that $\left[w_{h}, w_{h+1}\right] \subseteq U$ for all $h=1,2, \ldots, m-1$. (Note that this family of paths is nonempty, since $U$ is open and connected.)

Step 1: The domain of $f$ is nonempty.

We shall show, in particular, that $f\left(x_{0}\right)=0$. Since $f\left(x_{0}\right) \geq g\left(x_{0}, x_{0}\right)=0$, it suffices to show that $f\left(x_{0}\right) \leq 0$. To this end, let us suppose that $f\left(x_{0}\right)>0$ and take any $0<\varepsilon<f\left(x_{0}\right)$. By (17) we infer that for some closed polygonal path $\left[w_{h}\right]_{h=1}^{m}$ (with $\left.w_{1}=w_{m}=x_{0}\right)$ we have $\sum_{h=1}^{m-1} g\left(w_{h}, w_{h+1}\right)>\varepsilon$. Then according to (15), for any $\delta>0$ we can find a subdivision $\left\{x_{i}\right\}_{i=1}^{n}$ of the path $\left[w_{h}\right]_{h=1}^{n}$ (where $\left.x_{1}=x_{n}=x_{0}\right)$ and $\left\{x_{i}^{*}\right\}_{i=1}^{n}$ in $X^{*}$ such that $x_{i}^{*} \in T\left(x_{i}\right),\left\|x_{i+1}-x_{i}\right\|<\delta$ for all $i \geq 1$, and $\sum_{i=1}^{n-1}\left\langle x_{i}^{*}, x_{i+1}-x_{i}\right\rangle>\varepsilon$. Since $T$ is radially cyclically submonotone, we get a contradiction.

Step 2: $f(y) \geq f(x)+g(x, y), \forall(x, y) \in V$.

Take any $r<f(x)$ and choose a polygonal path $\left[w_{h}\right]_{h=1}^{m}$ in $U$ with $w_{1}=x_{0}$ and $w_{m}=x$ such that $\sum_{h=1}^{m-1} g\left(w_{h}, w_{h+1}\right)>r$. Set $w_{m+1}=y$. It follows from (17) that

$$
f(y) \geq \sum_{h=1}^{m} g\left(w_{h}, w_{h+1}\right)>r+g\left(w_{m}, w_{m+1}\right)=r+g(x, y) .
$$

Since $r<f(x)$ is arbitrarily close to $f(x)$, the proof is complete.

Step 3: $f(\bar{x})<+\infty$ for all $\bar{x} \in U$, and $f$ is locally Lipschitz on $U$.

Take any $\bar{x} \in U$ and choose a polygonal path $\left[w_{h}\right]_{h=1}^{m}$ in $U$ with $w_{1}=\bar{x}$ and $w_{m}=x_{0}$. It follows from Step 2 that for $y=x_{0}$ and for $x=w_{m-1}$, we have

$$
0=f\left(x_{0}\right) \geq f\left(w_{m-1}\right)+g\left(w_{m-1}, x_{0}\right),
$$

which shows that $f\left(w_{m-1}\right)$ is finite. Taking now $y=w_{m-1}$ and $x=w_{m-2}$, we conclude that $f\left(w_{m-2}\right)$ is finite. Proceeding like this, we finally conclude that $f(\bar{x})=f\left(w_{1}\right)<+\infty$. Now to show that $f$ is locally Lipschitz, given $\bar{x} \in U$ we take $x=y=\bar{x}$ and $\rho>0$ such that for any $x^{\prime}, y^{\prime} \in B_{\rho}(\bar{x})$ we have $\left(x^{\prime}, y^{\prime}\right) \in V$ and the estimate in (16). It follows from Step 2 that

$$
\left|f\left(y^{\prime}\right)-f\left(x^{\prime}\right)\right| \leq \max \left\{-g\left(x^{\prime}, y^{\prime}\right),-g\left(y^{\prime}, x^{\prime}\right)\right\},
$$

which yields that $f$ is locally Lipschitz on $U$.

Step 4:

$$
T(x) \subseteq \partial^{H} f(x) \quad \forall x \in U .
$$

Fix $x \in U$ and $x^{*} \in T(x)$. Let $r>0$ be such that $B_{r}(x) \subseteq U$. For every $u \in S_{X}$, we have by Step 2 that

$$
\left.\frac{f(x+t u)-f(x)}{t} \geq \frac{g(x, x+t u)}{t}, \text { for all } t \in\right] 0, r[.
$$

Since $f$ is locally Lipschitz, it suffices to show that for any $u \in S_{X}$ and any $\varepsilon>0$, there exists $\delta>0$ such that

$$
\inf _{0<t<\delta} \frac{g(x, x+t u)}{t} \geq\left\langle x^{*}, u\right\rangle-\varepsilon .
$$


From (14), we can associate to any $\varepsilon>0$ some $\delta>0$ (depending on $u$ ) such that for all $s \in] 0, \delta\left[\right.$ we can find $y^{*} \in T(x+s u)$ satisfying

$$
\left\langle y^{*}, u\right\rangle \geq\left\langle x^{*}, u\right\rangle-\varepsilon \text {. }
$$

Fix $0<t<\delta$. Given $\sigma>0$ and any subdivision $\left\{t_{i}\right\}_{i=1}^{n}$ of $[0, t]$ such that

$$
\sup _{i}\left(t_{i+1}-t_{i}\right)<\sigma
$$

we can find $x_{i}^{*} \in T\left(x+t_{i} u\right)$ such that

$$
\left\langle x_{i}^{*}, u\right\rangle \geq\left\langle x^{*}, u\right\rangle-\varepsilon .
$$

Setting $x_{i}:=x+t_{i} u$, we observe that $\left\{x_{i}\right\}_{i=1}^{n} \in S_{\sigma}(x, x+t u)$ and

$$
\begin{aligned}
\sum_{i=1}^{n-1}\left\langle x_{i}^{*}, x_{i+1}-x_{i}\right\rangle & =\sum_{i=1}^{n-1}\left\langle x_{i}^{*},\left(t_{i+1}-t_{i}\right) u\right\rangle \\
& \geq\left(\left\langle x^{*}, u\right\rangle-\varepsilon\right) \sum_{i=1}^{n-1}\left(t_{i+1}-t_{i}\right)=\left\langle x^{*}, u\right\rangle t-\varepsilon t .
\end{aligned}
$$

Since $\sigma>0$ can be taken arbitrarily small, we obtain

$$
g(x, x+t u) \geq\left\langle x^{*}, t u\right\rangle-t \varepsilon,
$$

and relation (19) follows for the $\delta$ introduced above.

Note that, if we assume in addition that $T$ is submonotone, then the above $\delta$ (in Step 4) does not depend on the direction $u \in S_{X}$; hence (19) yields $x^{*} \in \partial^{F} f(x)$.

Case 2 (general case): Let $U$ be an arbitrary nonempty open set. Then $U$ can be written as a disjoint union of open connected sets $U_{i}$. Applying the result of Case 1 for each $i$, we obtain a locally Lipschitz function $f_{i}$ on $U_{i}$ with $T(x) \subseteq \partial^{H} f(x)$ (resp. $T(x) \subseteq \partial^{F} f(x)$, if $T$ is submonotone), for all $x \in U_{i}$. Define $f: U \rightarrow \mathbb{R}$ by $f(x)=f_{i(x)}(x)$, where $i(x)$ is the unique index such that $x \in U_{i(x)}$. It follows that $f$ is locally Lipschitz and $T \subseteq \partial^{H} f$ (resp. $T \subseteq \partial^{F} f$ ).

\section{Cyclic submonotonicity}

In Theorem 4 we obtained a sufficient condition ensuring that an operator $T$ is included in the subdifferential $\partial^{C} f$ of a locally Lipschitz function $f$. In this section we reinforce Definition 3 (by using a notion of approximate subdivisions of closed polygonal paths) to ensure the coincidence of $T$ with the subdifferential $\partial^{C} f$. This leads to a notion of cyclic submonotonicity, which turns out (in Section 5) to be a necessary and sufficient condition for the integration process described in Theorem 4 Its relation with radial cyclic submonotonicity is given in Proposition 17.

Let us first give the definition of a $\delta$-subdivision of a closed polygonal path.

Definition 5. Given $\delta>0$ and a closed polygonal path $\left[w_{h}\right]_{h=1}^{m}$, we say that $\left\{x_{i}\right\}_{i=1}^{n}$ is a $\delta$-subdivision of $\left[w_{h}\right]_{h=1}^{m}$ if $x_{n}=x_{1}$ and

(i) $\left\{x_{i}\right\}_{i=1}^{n} \subseteq B_{\delta}\left(\left[w_{h}\right]_{h=1}^{m}\right)$,

(ii) $\left\|x_{i+1}-x_{i}\right\|<\delta$, for $i \in \mathbb{N}_{n-1}$, and

(iii) there exists a finite sequence $\left\{k_{h}\right\}_{h=1}^{m}$ with $1=k_{1}<k_{2}<\ldots<k_{m}:=n$ such that for $1 \leq h \leq m-1$ we have

$$
k_{h} \leq i<k_{h+1} \Longrightarrow\left\|\frac{x_{i+1}-x_{i}}{\left\|x_{i+1}-x_{i}\right\|}-\frac{w_{h+1}-w_{h}}{\left\|w_{h+1}-x_{h}\right\|}\right\|<\delta .
$$

We are now ready to give the following definition. 
Definition 6. An operator $T$ is called cyclically submonotone, if for any closed polygonal path $\left[w_{h}\right]_{h=1}^{m}$ and any $\varepsilon>0$, there exists $\delta>0$ such that for all $\delta$ subdivisions $\left\{x_{i}\right\}_{i=1}^{n}$ of $\left[w_{h}\right]_{h=1}^{m}$ and all $x_{i}^{*} \in T\left(x_{i}\right)$, one has

$$
\sum_{i=1}^{n-1}\left\langle x_{i}^{*}, x_{i+1}-x_{i}\right\rangle \leq \varepsilon \sum_{i=1}^{n-1}\left\|x_{i+1}-x_{i}\right\| .
$$

If $U$ is an open subset of $X$, an operator $T$ is said to be cyclically submonotone on $U$ if (21) holds for closed polygonal paths and $\delta$-subdivisions in $U$. Furthermore, a cyclically submonotone operator $T$ on $U$ is called maximal cyclically submonotone on $U$, if there is no cyclically submonotone operator $S \neq T$ such that $T(x) \subseteq S(x)$ for all $x \in U$.

Let us note that, as follows from (12), the length of a subdivision of a path is always equal to the length of the initial path. On the contrary, the definition of a $\delta$ subdivision is more general, since no direct constraint on its length is imposed. This flexibility in Definition 5 enables us to show that, unlike the case of radial cyclic submonotonicity, every cyclically submonotone operator is also ds-submonotone.

Proposition 7. Every cyclically submonotone operator on $U$ is ds-submonotone on $U$. Consequently, if $U \subseteq \operatorname{int} \operatorname{dom}(T)$, then $T$ is also locally bounded on $U$.

Proof. Let $x_{0} \in U, \varepsilon>0$ and $e \in S_{X}$. Since $U$ is open, there exists $\lambda>0$ such that $\left[x_{0}, x_{0}+\lambda e\right] \subseteq U$. Let $m=3, w_{1}=x_{0}=w_{3}$ and $w_{2}=x_{0}+\lambda e$. For $\varepsilon>0$ and for the path $\left[w_{h}\right]_{h=1}^{3}$, take $\delta>0$ as in Definition 6, and set $\delta^{\prime}=\delta / 2$. Then if $x_{1}, x_{2} \in B_{\delta^{\prime}}\left(x_{0}\right)$ are such that $x_{1} \neq x_{2}$ and $\left\|\frac{x_{1}-x_{2}}{\left\|x_{1}-x_{2}\right\|}-e\right\|<\delta^{\prime}$, we can easily check that for $x_{3}=x_{1},\left\{x_{i}\right\}_{i=1}^{3}$ is a $\delta$-subdivision of the path $\left[w_{h}\right]_{h=1}^{3}$. So relation (21) yields (11), and $T$ is ds-submonotone at $x_{0}$. Since $x_{0}$ is arbitrary in $U$, it follows that $T$ is ds-submonotone on $U$. The last assertion follows from [11, Theorem $2.4]$.

Remarks. 1. Every cyclically submonotone operator is radially cyclically submonotone on every open subset $U$ of its domain. Indeed, if (21) is true and $\left\{x_{i}\right\}_{i=1}^{n}$ is a subdivision of $\left[w_{h}\right]_{h=1}^{m}$ in $U$, then $\sum_{i=1}^{n-1}\left\|x_{i+1}-x_{i}\right\|=\sum_{i=1}^{n-1}\left\|w_{i+1}-w_{i}\right\|$. It follows that (21) yields (13) for $\varepsilon^{\prime}=\varepsilon\left(\sum_{i=1}^{n-1}\left\|w_{i+1}-w_{i}\right\|\right)^{-1}$.

2. It is obvious that every cyclically monotone operator is cyclically submonotone. On the other hand, an operator can even be strongly monotone, without being cyclically submonotone, as one can see from the example (also used in [9] for a similar purpose) of the operator $T: \mathbb{R}^{2} \rightarrow \mathbb{R}^{2}$, with

$$
T(x, y)=\left\langle\frac{x}{2}-y, x+\frac{y}{2}\right\rangle .
$$

3. Cyclic submonotonicity is a separably determined property, i.e., an operator $T: X \rightrightarrows X^{*}$ is cyclically submonotone on $U$ if, and only if, for every separable closed subspace $Y$ of $X$, the operator $\left.T\right|_{Y}: Y \rightrightarrows Y^{*}$ (defined for all $y_{1}, y_{2} \in Y$ by $\left.\left\langle\left. T\right|_{Y}\left(y_{1}\right), y_{2}\right\rangle:=\left\langle T\left(y_{1}\right), y_{2}\right\rangle\right)$ is cyclically submonotone on $U \cap Y$.

We now show that if $T$ is locally bounded and cyclically submonotone (resp. ds-submonotone) on $U$, then so is the $w^{*}$-cusco generated by $T$. Let us recall that if $U$ is an open subset of $\operatorname{dom}(T)$, the local boundedness assumption on $T$ becomes superfluous, since it follows from its ds-submonotonicity (see Proposition [7). 
Proposition 8. If $T$ is locally bounded and cyclically submonotone (resp. ds-submonotone) on $U$, then the operators $\bar{T}$ and $\overline{c o}^{w^{*}}(T)$ are also cyclically submonotone (resp. ds-submonotone) on $U$. In particular, the $w^{*}$-cusco generated by $T$ is cyclically submonotone (resp. ds-submonotone) on $U$.

Proof. It is easily seen from (21) that $\overline{c o} w^{*}(T)$ is cyclically submonotone. In order to prove that $\bar{T}$ is cyclically submonotone, let us consider a closed polygonal path $\left[w_{h}\right]_{h=1}^{m} \subseteq U$ and $\varepsilon>0$. Let us take $\delta>0$ guaranteed by Definition 6 for the operator $T$, and let us consider any $\delta$-subdivision $\left\{x_{i}\right\}_{i=1}^{n}$ of $\left[w_{h}\right]_{h=1}^{m}$ in $U$ and $x_{i}^{*} \in \bar{T}\left(x_{i}\right)$. Then there exist nets $\left(x_{i}(\lambda)\right)_{\lambda \in \Lambda}$ and $\left(x_{i}^{*}(\lambda)\right)_{\lambda \in \Lambda}$ such that $x_{i}^{*}(\lambda) \in$ $T\left(x_{i}(\lambda)\right),\left(x_{i}(\lambda)\right) \stackrel{\|\cdot\|}{\longrightarrow} x_{i}$ and $\left(x_{i}^{*}(\lambda)\right) \stackrel{w^{*}}{\longrightarrow} x_{i}^{*}$. Since $\left\{x_{i}\right\}_{i=1}^{n}$ is a finite sequence, there exists $\lambda_{0} \in \Lambda$ such that $\left\{x_{i}(\lambda)\right\}_{i=1}^{n}$ is a $\delta$-subdivision of $\left[w_{h}\right]_{h=1}^{m}$ for all $\lambda \succeq \lambda_{0}$ (where $\succeq$ is the preorder relation of $\Lambda$ ). It follows from (21) that

$$
\sum_{i=1}^{n}\left\langle x_{i}^{*}(\lambda), x_{i+1}(\lambda)-x_{i}(\lambda)\right\rangle \leq \varepsilon \sum_{i=1}^{n}\left\|x_{i+1}(\lambda)-x_{i}(\lambda)\right\| .
$$

Taking limits on both sides, we obtain

$$
\sum_{i=1}^{n}\left\langle x_{i}^{*}, x_{i+1}-x_{i}\right\rangle \leq \varepsilon \sum_{i=1}^{n}\left\|x_{i+1}-x_{i}\right\| .
$$

This shows that $\bar{T}$ is cyclically submonotone; hence so is the operator $\overline{c o}^{w^{*}}(\bar{T})$. Since now $T$ is locally bounded on $U$, we have

$$
\bar{T}(x):=\bigcap_{\varepsilon>0} \overline{\left\{T\left(x^{\prime}\right): x^{\prime} \in B_{\varepsilon}(x) \cap \operatorname{dom}(T)\right\}} w^{*}
$$

for all $x \in U$. Using Proposition 1, we conclude that $\overline{c o} w^{*}(\bar{T})$ is the minimal $w^{*}$ cusco operator containing $T$. This finishes the proof. The assertions concerning ds-submonotonicity can be proved likewise.

The following proposition reveals an important feature of cyclic submonotonicity.

Proposition 9. Let $U$ be an open subset of $\operatorname{dom}(T)$, and $T$ a cyclically submonotone operator on $U$. The following statements are equivalent:

(i) $T$ is $w^{*}$-cusco on $U$;

(ii) $T$ is minimal $w^{*}$-cusco on $U$;

(iii) $T$ is maximal ds-submonotone on $U$;

(iv) $T$ is maximal cyclically submonotone on $U$.

Proof. Implication (ii) $\Rightarrow(\mathrm{i})$ is obvious. Assume now that (i) holds. Using Proposition 7 we conclude that $T$ is ds-submonotone on $U$. Since $T$ is $w^{*}$-cusco, from [11. Lemma 3.2] it follows that $T$ is maximal ds-submonotone on $U$. Hence (i) $\Rightarrow($ iii).

(iii) $\Rightarrow$ (iv): Let $S$ be a cyclically submonotone operator whose graph contains the graph of $T$. Then $S$ is ds-submonotone (see Proposition 17); hence it coincides with $T$.

(iv) $\Rightarrow$ (ii): Since $T$ is locally bounded on $U \subseteq \operatorname{int} \operatorname{dom}(T)$, Proposition 8 guarantees that $T$ is $w^{*}$-cusco. Assume that there exists $S \subseteq T$ such that $S$ is $w^{*}$-cusco. Obviously $S$ will also be cyclically submonotone. Since (i) $\Rightarrow($ iv),$S$ is maximal cyclically submonotone, whence $S=T$. 
Remark 10. We recall from [23] that if $X$ is Asplund (resp. $X$ has a Gâteaux differentiable norm), then every minimal $w^{*}$-cusco operator is single-valued and $(\|\cdot\|-\|\cdot\|)$ upper semicontinuous (resp. $\left(\|\cdot\|-w^{*}\right)$ upper semicontinuous) at every point of a $G_{\delta}$ dense set.

Corollary 11. Let $T$ be a cyclically submonotone operator on an open subset $U$. Then $\overline{c o}^{w^{*}}(T)$ is maximal cyclically submonotone on $U$.

Proof. Since $T$ is locally bounded, the operator $S:=\overline{c o}^{w^{*}}(\bar{T})$ is $w^{*}$-cusco. By Proposition 8, $S$ is also cyclically monotone. The conclusion follows from Proposition 9 .

Let $Z$ be a closed subspace of $X$ and define the multivalued operator $S: Z \rightrightarrows Z^{*}$ as follows:

$$
S(z)=\left\{z^{*} \in Z^{*}: \exists x^{*} \in T(z) \text { such that } z^{*}=\left.x^{*}\right|_{Z}\right\}
$$

(where $\left.x^{*}\right|_{Z}$ denotes the restriction of the functional $x^{*}$ to $Z$ ).

Lemma 12. (i) If $T$ is locally bounded and $w^{*}$-cusco on an open subset $U$, then $S$ is $\sigma\left(Z^{*}, Z\right)$-cusco on $U \cap Z$, where $\sigma\left(Z^{*}, Z\right)$ denotes the $w^{*}$-topology on $Z^{*}$.

(ii) If $T$ is maximal cyclically submonotone on $U$ and if $U \subseteq \operatorname{intdom}(T)$, then $S$ is maximal cyclically submonotone on $U \cap Z$.

Proof. Assertion (i) follows easily since $S$ has a $\sigma\left(Z^{*}, Z\right)$-closed graph on $U \cap Z$. To show (ii), let us observe (from Definition 6 ) that if $T$ is cyclically submonotone on $U$, then $S$ is cyclically submonotone on $U \cap Z$. By Propositions 7 and 9 we have that $T$ is locally bounded and $w^{*}$-cusco. It follows by (i) that $S$ is $\sigma\left(Z^{*}, Z\right)$-cusco on $U \cap Z$; so the proof finishes by a new application of Proposition 9 (i) $\rightarrow$ (iv).

\section{MAin RESUlts}

Throughout this section $U$ will always denote a nonempty open subset of $X$. Let us give the following definition.

Definition 13. A locally Lipschitz function $f: U \rightarrow \mathbb{R}$ is called subsmooth if $\partial^{C} f$ is ds-submonotone on $U$.

Every subsmooth function $f$ is regular (see [24], [11, Theorem 4.1]), a locally Lipschitz function $f$ being called regular ([6]) if $f^{\prime}(x, d)=f^{o}(x, d)$ for all $d \in X$. It follows that

$$
\partial^{C} f=\partial^{H} f
$$

In the particular case where $X$ has some regularity, subsmoothness is characterized as follows.

Proposition 14. Let $X$ be an Asplund space (resp. X has a Gâteaux differentiable renorming). Then a locally Lipschitz function $f: U \rightarrow \mathbb{R}$ is subsmooth if, and only if, $\partial^{F} f\left(\right.$ resp. $\left.\partial^{H} f\right)$ is ds-submonotone on $U$.

Proof. The "necessity" part is obvious. To show the "sufficiency" part, let $T=\partial^{F} f$ (resp. $\partial^{H} f$ ). Since $f$ is locally Lipschitz and $T$ is included in $\partial^{C} f$, it follows that $T$ is locally bounded. Using Corollary 2 and Proposition [8, we obtain that $\partial^{C} f$ is ds-submonotone, hence that $f$ is subsmooth. (Note that this implies that $\operatorname{dom}\left(\partial^{H} f\right)=U$.) 
It follows from Definition 13 and the comments after the definition of ds-submonotonicity (in Section 2) that every convex or continuously differentiable (i.e., $C^{1}$ ) function is subsmooth. One of the main results in [24] is the following characterization of subsmooth functions in finite dimensions: a (locally Lipschitz) function $f$ on $U \subseteq \mathbb{R}^{n}$ is subsmooth if, and only if, it is lower $C^{1}$, a function $f$ being called lower $C^{1}$ if for each $x_{0} \in U$, there exist a neighborhood $V$ of $x_{0}$, a compact set $S$ and a jointly continuous function $g: V \times S \rightarrow \mathbb{R}$ such that, for all $x \in V$, $f(x)=\max _{s \in S} g(x, s)$ and $D_{x} g$ (exists and) is jointly continuous. In the last section, we will give some typical examples of subsmooth functions in infinite dimensions.

We now state the main results of the paper.

Theorem A. For a locally Lipschitz function $f: U \rightarrow \mathbb{R}$, the following are equivalent:

(i) $f$ is subsmooth;

(ii) $\partial^{C} f$ is maximal cyclically submonotone on $U$.

Theorem B. Let $U$ be an open connected subset of $X$, and $f_{1}, f_{2}$ two subsmooth (or, more generally, regular) functions on $U$ such that $\partial^{C} f_{1}=\partial^{C} f_{2}$. Then $f_{1}=$ $f_{2}+c$ for some $c \in \mathbb{R}$.

Theorem C. If $T: X \rightrightarrows X^{*}$ is a multivalued operator and $U$ an open subset of $\operatorname{dom}(T)$, then $T$ is maximal cyclically submonotone on $U$ if, and only if, $T=$ $\partial^{C} f=\partial^{H} f$ for some subsmooth function $f: U \rightarrow \mathbb{R}$, which is unique (up to a constant) on every connected subset of $U$. If, in addition, $T$ is submonotone, then $T=\partial^{F} f$.

Proof of Theorem A. The implication (ii) $\Rightarrow(\mathrm{i})$ is clear in view of Proposition 7 and Definition 13.

For the implication (i) $\Rightarrow$ (ii), set $T:=\partial^{C} f$. Since $T$ is $w^{*}$-cusco, in view of Proposition 9 (i) $\Rightarrow$ (iv), it clearly suffices to show that $T$ is cyclically submonotone. To this end, consider any closed polygonal path $\left[w_{h}\right]_{h=1}^{m} \subseteq U$ and any $\varepsilon>0$. Set $C=\left[w_{h}\right]_{h=1}^{m}$ and

$$
e_{h}=\frac{w_{h+1}-w_{h}}{\left\|w_{h+1}-w_{h}\right\|}
$$

for $h \in \mathbb{N}_{m-1}:=\{1,2, \ldots, m-1\}$.

Since $T$ is ds-submonotone, it follows that for every $x \in C$ and $h \in \mathbb{N}_{m-1}$, there exists $\alpha(x, h)>0$ such that

$$
\frac{\left\langle x_{1}^{*}-x_{2}^{*}, x_{2}-x_{1}\right\rangle}{\left\|x_{1}-x_{2}\right\|}<\varepsilon
$$

whenever $x_{1} \neq x_{2}$ with $\left\|x_{i}-x\right\|<\alpha(x, h), x_{i}^{*} \in T\left(x_{i}\right)(i=1,2)$ and

$$
\left\|\frac{x_{1}-x_{2}}{\left\|x_{1}-x_{2}\right\|}-e_{h}\right\|<\alpha(x, h) \text {. }
$$

Set $\beta(x):=\min _{h \in \mathbb{N}_{m}} \alpha(x, h)$, and note that (24) holds for all $x_{1} \neq x_{2}$ such that $x_{i} \in$ $B_{\beta(x)}(x)(i=1,2)$ and $\frac{x_{1}-x_{2}}{\left\|x_{1}-x_{2}\right\|} \in \bigcup_{h \in \mathbb{N}_{m-1}} B_{\beta(x)}\left(e_{h}\right)$.

Let $\delta>0$ be a Lebesgue number of the open covering $\left(B_{\beta(x)}(x)\right)_{x \in C}$ of the compact set $C$, i.e.,

$$
\forall w \in C, \exists x \in C: B_{\delta}(w) \subseteq B_{\beta(x)}(x)
$$


Let us consider any $\delta$-subdivision $\left\{x_{i}\right\}_{i=1}^{n}$ of $C=\left[w_{h}\right]_{h=1}^{m}$. Since $f$ is locally Lipschitz, using Lebourg's mean value theorem ([13]) on every segment $\left[x_{i}, x_{i+1}\right]$ (for $\left.i \in \mathbb{N}_{n-1}\right)$, we infer the existence of $\left.z_{i} \in\right] x_{i}, x_{i+1}\left[\right.$ and $z_{i}^{*} \in T\left(z_{i}\right)$ such that

$$
f\left(x_{i+1}\right)-f\left(x_{i}\right)=\left\langle z_{i}^{*}, x_{i+1}-x_{i}\right\rangle .
$$

Adding the above equalities, we have

$$
\sum_{i=1}^{n-1}\left\langle z_{i}^{*}, x_{i+1}-x_{i}\right\rangle=0,
$$

which yields

$$
\sum_{i=1}^{n-1}\left\langle x_{i}^{*}, x_{i+1}-x_{i}\right\rangle=\sum_{i=1}^{n-1}\left\langle x_{i}^{*}-z_{i}^{*}, x_{i+1}-x_{i}\right\rangle .
$$

Since $\left\{x_{i}\right\}_{i=1}^{n}$ is a $\delta$-subdivision of $C$, it follows from (24) and (25) that for every $i \in \mathbb{N}_{n-1}$,

$$
\left\langle x_{i}^{*}-z_{i}^{*}, \frac{x_{i+1}-x_{i}}{\left\|x_{i+1}-x_{i}\right\|}\right\rangle=\left\langle x_{i}^{*}-z_{i}^{*}, \frac{z_{i}-x_{i}}{\left\|z_{i}-x_{i}\right\|}\right\rangle<\varepsilon,
$$

which, combined with (27), yields

$$
\sum_{i=1}^{n-1}\left\langle x_{i}^{*}, x_{i+1}-x_{i}\right\rangle=\varepsilon \sum_{i=1}^{n-1}\left\|x_{i+1}-x_{i}\right\| .
$$

This finishes the proof.

Before proceeding to the proof of Theorem B, we need the following easy result.

Lemma 15. Let $f: U \rightarrow \mathbb{R}$ be a locally Lipschitz function, let $Z$ be a closed linear subspace of $X$, and consider the function $g: Z \cap U \rightarrow \mathbb{R}$ defined by

$$
g(z)=f(z) \text { for all } z \in U \cap Z .
$$

If $S: Z \rightrightarrows Z^{*}$ is as in (22) for $T=\partial^{H} f$, then we have:

(i) $S(z) \subseteq \partial^{H} g(z)$ for all $z \in U \cap Z$.

(ii) If for some $x_{0} \in U \cap Z$ we have $\partial^{H} f\left(x_{0}\right)=\partial^{C} f\left(x_{0}\right)$, then $S\left(x_{0}\right)=\partial^{H} g\left(x_{0}\right)=$ $\partial^{C} g\left(x_{0}\right)$.

Proof. (i) Let $z \in U \cap Z$ and $z^{*} \in S(z)$. Then $z^{*}=\left.x^{*}\right|_{Z}$ for some $x^{*} \in \partial^{H} f(z)$. Since $f$ is locally Lipschitz, by (5) we conclude that $g^{\prime}(z, u)=f^{\prime}(z ; u) \geq\left\langle x^{*}, u\right\rangle$, for all $u \in Z$. It follows that $z^{*} \in \partial^{H} g(z)$.

(ii) Suppose now that for some $x_{0} \in U \cap Z$ we have $\partial^{H} f\left(x_{0}\right)=\partial^{C} f\left(x_{0}\right)$. By (i) we have $S\left(x_{0}\right) \subseteq \partial^{H} g\left(x_{0}\right) \subseteq \partial^{C} g\left(x_{0}\right)$. Let us show that $\partial^{C} g\left(x_{0}\right) \subseteq S\left(x_{0}\right)$. Indeed, let $z_{0}^{*} \in \partial^{C} g\left(x_{0}\right)$. Then from (4) we have

$$
\left\langle z_{0}^{*}, u\right\rangle \leq g^{o}\left(x_{0} ; u\right) \leq f^{o}\left(x_{0} ; u\right), \text { for all } u \in Z \text {. }
$$

Using the Hahn-Banach theorem, we conclude the existence of some $x^{*} \in X^{*}$ such that $\left.x^{*}\right|_{Z}=z^{*}$ and $\left\langle x^{*},.\right\rangle \leq f^{o}\left(x_{0} ;.\right)$, so that $x^{*} \in \partial^{C} f\left(x_{0}\right)=\partial^{H} f\left(x_{0}\right)$. It follows that $z^{*} \in S\left(x_{0}\right)$.

Proof of Theorem B. Suppose that $f_{1}, f_{2}$ are two subsmooth functions on $U$ such that $\partial^{C} f_{1}=\partial^{C} f_{2}$. Without loss of generality, we suppose that $0 \in U$. For any 
$x \in X$, let us set $Z=\operatorname{span}[x], g_{i}=\left.f_{i}\right|_{Z}$ (the restriction of $f_{i}$ to $\left.Z, i=1,2\right)$ and $T=\partial^{H} f_{1}$. By (23) we have

$$
T=\partial^{H} f_{1}=\partial^{C} f_{1}=\partial^{C} f_{2}=\partial^{H} f_{2},
$$

and by Lemma 15(ii),

$$
\partial^{H} g_{1}=\partial^{C} g_{1}=\partial^{C} g_{2}=\partial^{H} g_{2}
$$

Since $Z$ is a one-dimensional space (in fact, separable would suffice), it follows from 3. Theorem 5.12] (see also [5, Section 4.2]) that the regular functions $g_{1}, g_{2}$ are essentially smooth.

Case 1: Suppose that $U$ is convex.

Then the set $U \cap Z$ is connected; so relation (29) yields $g_{1}=g_{2}+c$ for some $c \in \mathbb{R}$ (see [5, Proposition 4.12] or [3, Proposition 5.9]). Since $g_{i}=\left.f_{i}\right|_{Z}$, we obtain

$$
f_{1}(x)-f_{2}(x)=c=f_{1}(0)-f_{2}(0) .
$$

Since $x$ is arbitrarily chosen, we obtain $f_{1}=f_{2}+c$ on $U$.

Case 2 (general): Since $U$ is open and $X$ is locally convex, from case 1 we conclude that $f_{1}-f_{2}$ is locally constant on $U$. Since now $U$ is connected, it follows that $f_{1}-f_{2}$ is constant on $U$. The proof is complete.

Let us now proceed to the proof of Theorem C. We shall need the following lemma.

Lemma 16. Let $f: U \rightarrow \mathbb{R}$ be locally Lipschitz, $x_{0} \in X$, and let $Y$ be a separable subspace of $X$. Then there exists a separable subspace $Z$ of $X$ containing $Y$ and $x_{0}$ such that for the function $g: Z \cap U \rightarrow \mathbb{R}$ given by $g=\left.f\right|_{U \cap Z}($ as in (28)) we have

$$
g^{o}\left(x_{0} ; u\right)=f^{o}\left(x_{0} ; u\right) \text { for all } u \in Z,
$$

and consequently

$$
\partial^{C} g\left(x_{0}\right)=\left\{z^{*} \in Z^{*}: z^{*}=\left.x^{*}\right|_{Z}, z^{*} \in \partial^{C} f\left(x_{0}\right)\right\} .
$$

Proof. Let $Y_{0}=\operatorname{span}\left[Y, x_{0}\right]$ be the closed linear space generated by $Y$ and $\left\{x_{0}\right\}$, and let $D_{0}$ be a countable dense subset of $Y_{0}$. Then for every $d \in D_{0}$, there exist $\left\{x_{n}\right\}_{n \geq 1}$ in $X$ and $\left\{t_{n}\right\}_{n \geq 1}$ in $] 0,1\left[\operatorname{such}\right.$ that $\left(x_{n}\right) \longrightarrow x_{0},\left(t_{n}\right) \rightarrow 0^{+}$and

$$
f^{o}\left(x_{0} ; d\right)<\frac{f\left(x_{n}+t_{n} d\right)-f\left(x_{n}\right)}{t_{n}}+\frac{1}{n} .
$$

Set $A_{0}(d)=\left\{x_{n}: n \in \mathbb{N}\right\}$ and $A_{0}=\bigcup_{d \in D_{0}} A_{0}(d)$. Consider the separable space $Y_{1}=\operatorname{span}\left[Y_{0}, D_{0}\right]$, let $D_{1}$ be a countable dense subset of $Y_{1}$ and define (using (32) $A_{1}(d)$ for all $d$ in $D_{1}$ as above and $A_{1}=\bigcup_{d \in D_{1}} A(d)$. Proceeding like this, we obtain an increasing sequence of closed separable subspaces $Y_{n}$ of $X$ and a sequence $\left(D_{n}\right)$ of countable subsets such that $D_{n}$ is dense in $Y_{n}$. Set

$$
Z=\bigcup_{n} Y_{n}
$$

and $g=\left.f\right|_{Z}$. Then for any $u \in Z$ and $\varepsilon>0$ there exist $n \in \mathbb{N}$ and $d \in D_{n}$ such that $\|u-d\|<\varepsilon$. Using (32), we conclude easily that $g^{o}\left(x_{0} ; d\right)=f^{o}\left(x_{0} ; d\right)$. Since the functions $u \mapsto g^{o}\left(x_{0} ; u\right)$ and $u \mapsto f^{o}\left(x_{0} ; u\right)$ are Lipschitz, (30) follows. Relation (31) is now an easy consequence of (30) and the Hahn-Banach theorem. 
Proof of Theorem C. The sufficiency part following from Theorem A, we only have to show the necessity part.

To this end, let us suppose that $T$ is maximal cyclically submonotone on $U$. In particular, $T$ is a locally bounded ds-submonotone and radially cyclically submonotone operator on $U$ (see Proposition 7 and Remark 1). It follows by Theorem 4 that $T(x) \subseteq \partial^{H} f(x)$ for all $x \in U$, where $f$ is given by (17). Let us show that $T(x)=\partial^{C} f(x)$ for all $x \in U$.

Suppose that the contrary holds. Then for some $x_{0} \in X$ and $x_{0}^{*} \in \partial f^{C}\left(x_{0}\right)$, we have $x_{0}^{*} \notin T\left(x_{0}\right)$. By Proposition 9. $T\left(x_{0}\right)$ is a nonempty $w^{*}$-closed convex set; hence there exists $u \in X$ such that

$$
\left\langle x_{0}^{*}, u\right\rangle>\sup _{x^{*} \in T\left(x_{0}\right)}\left\langle x^{*}, u\right\rangle .
$$

Set $Y=\operatorname{span}\left[x_{0}, u\right]$, and consider the separable subspace $Z$ of $X$ given by Lemma 16 and $g=\left.f\right|_{Z}$. Let $S: Z \rightrightarrows Z^{*}$ be as in (22), i.e., for every $z \in U \cap Z$,

$$
S(z)=\left\{z^{*} \in Z^{*}: z^{*}=\left.x^{*}\right|_{Z} \text { for some } x^{*} \in T(z)\right\} .
$$

Then, by Lemma 12(i), $S$ is $\sigma\left(Z^{*}, Z\right)$-cusco on $U \cap Z$, and, by Lemma 15)(i),

$$
S \subseteq \partial^{H} g .
$$

Since $S$ has nonempty values on $U \cap Z$, the above relation yields that $S(x)=$ $\left\{D^{H} g(x)\right\}$ for all points $x$ for which the Hadamard derivative $D^{H} g(x)$ exists. Since $Z$ is a separable Banach space, it admits a Gâteaux smooth renorming. It follows that the Clarke subdifferential $\partial^{C} g$ is given by (7) and is the smallest $\sigma\left(Z^{*}, Z\right)$ cusco mapping whose graph contains the graph of the Hadamard derivative $D^{H} g$. Since $S$ is $\sigma\left(Z^{*}, Z\right)$-cusco, it follows that $\operatorname{Gr}\left(\partial^{C} g\right) \subseteq \operatorname{Gr}(S)$, for all $x \in U$. Since $\operatorname{Gr}\left(\partial^{H} g\right) \subseteq \operatorname{Gr}\left(\partial^{C} g\right)$, we conclude that

$$
S=\partial^{H} g=\partial^{C} g \text { on } U \cap Z \text {. }
$$

In particular, $S\left(x_{0}\right)=\partial^{C} g\left(x_{0}\right)$, and, using the conclusion of Lemma 16 .

$$
S\left(x_{0}\right)=\left\{z^{*} \in Z^{*}: z^{*}=\left.x^{*}\right|_{Z}, x^{*} \in \partial^{C} f\left(x_{0}\right)\right\} .
$$

Let $z_{0}^{*}:=\left.x_{0}^{*}\right|_{Z} \in S\left(x_{0}\right)=\partial^{C} g\left(x_{0}\right)$. Since $u \in Z$, it follows that

$$
\left\langle x_{0}^{*}, u\right\rangle=\left\langle z_{0}^{*}, u\right\rangle \leq g^{o}\left(x_{0} ; u\right)=f^{o}\left(x_{0} ; u\right) .
$$

This yields a contradiction to (33), since

$$
g^{o}\left(x_{0} ; u\right)=\sup _{z^{*} \in \partial^{C} g\left(x_{0}\right)}\left\langle z^{*}, u\right\rangle=\sup _{x^{*} \in S\left(x_{0}\right)}\left\langle x^{*}, u\right\rangle=\sup _{x^{*} \in T\left(x_{0}\right)}\left\langle x^{*}, u\right\rangle .
$$

Hence we have shown that $T=\partial^{C} f$ on $U$. It follows from Definition 13 that $f$ is subsmooth, and by Theorem B that it is unique (modulo a constant) in every connected subset of $U$.

If moreover $T$ is submonotone, then, using Theorem 4 again, we infer that $T(x) \subseteq$ $\partial^{F} f(x)$, for all $x \in U$. Hence $\operatorname{Gr}(T) \subseteq \operatorname{Gr}\left(\partial^{F} f\right) \subseteq \operatorname{Gr}\left(\partial^{C} f\right)$ and $T=\partial^{C} f$ (on $U$ ), whence $T=\partial^{F} f=\partial^{C} f$ on $U$.

An inspection of the above proof yields the following result.

Proposition 17. Suppose that $T$ is a locally bounded $w^{*}$-cusco operator on an open subset $U$ of $X$. Then $T$ is (maximal) cyclically submonotone on $U$ if, and only if, $T$ is radially cyclically submonotone and ds-submonotone on $U$. 
Proof. If $T$ is cyclically submonotone, then from Proposition 7 and Remark 1, it follows that $T$ is radially cyclically submonotone and ds-submonotone on $U$. Conversely, if $T$ is radially cyclically submonotone and ds-submonotone on $U$, then by Theorem 4 we infer that $T \subseteq \partial^{H} f$ on $U$ for some locally Lipschitz function $f$.

Since now $T$ is locally bounded and $w^{*}$-cusco on $U$, by Lemma 12 (i), for every closed subspace $Z$ of $X$, the operator $S$ given in (22) is $\sigma\left(Z^{*}, Z\right)$-cusco on $U \cap Z$. Thus, repeating the arguments of the above proof, we obtain that $T=\partial^{C} f$ on $U$ and that $f$ is subsmooth. It follows from Theorem $\mathrm{A}$ that $T$ is (maximal) cyclically submonotone on $U$.

\section{EXAMPLES OF SUBSMOOTH FUNCTIONS}

Apart from the classes of convex, continuous, or $C^{1}$ functions (or of sums of such functions), typical examples of subsmooth functions include certain types of marginal functions, as for instance the class of lower $C^{1}$ functions introduced in 24] (and also considered in [21] and [16]). Let us note that subdifferentiability properties of marginal functions have been studied by many authors; see for instance [3], 7], 8] and [11.

In the sequel let $A$ be an arbitrary nonempty set and $U$ an open subset of $X$. We consider the marginal function $f: U \rightarrow \mathbb{R}$ defined for every $x \in U$ by

$$
f(x)=\sup _{\alpha \in A} g(x, \alpha),
$$

where $g: U \times A \rightarrow \mathbb{R}$ is such that $g(\cdot, \alpha)$ is a regular locally Lipschitz function and $f(x)<+\infty$ for every $x \in U$. Let us also make the following assumptions.

(i) For every $x_{0} \in U$, there exists $\delta>0$ such that the set

$$
\bigcup\left\{\partial^{C} g(x, \alpha): \alpha \in A, x \in B_{\delta}\left(x_{0}\right), g(x, \alpha) \geq f\left(x_{0}\right)-\delta\right\}
$$

is norm bounded.

(ii) For every $x \in X$ and $e \in S_{X}$ there is an $\varepsilon>0$ such that for every $\gamma>0$ there exists $\delta>0$ such that

$$
f^{\prime}(y, a ; e)-f^{\prime}(x, a ; e)<\gamma
$$

whenever $\|x-y\|<\delta$ and $g(x, \alpha) \geq f(x)-\varepsilon$.

(iii) For every $x_{0} \in U, e \in S_{X}$ and $\varepsilon>0$, there exists $\delta>0$ such that

$$
g^{\prime}(x, a ; e)-\frac{g(x+t e, a)-g(x, a)}{t}<\varepsilon
$$

for all $x \in B_{\delta}\left(x_{0}\right)$, all $\left.t \in\right] 0, \delta\left[\right.$ and all $a \in A$ with $g(x, a) \geq f\left(x_{0}\right)-\delta$.

The following result is an easy consequence of results established in [11.

Theorem 18. If $f$ is a marginal function (relation (34)) satisfying conditions (i)(iii), then $f$ is subsmooth on $U$ and $\partial^{H} f=\partial^{C} f$ is maximal cyclically submonotone on $U$.

Proof. By 11, Theorem 5.4 (a)], $f$ is locally Lipschitz, and by [11, Lemma 5.3] and [11, Theorem $5.2(\mathrm{~d})], f$ is regular; therefore $\partial^{H} f=\partial^{C} f$. Now again by [11] Theorem 5.4 (a)], $\partial^{C} f$ is ds-submonotone in $U$, i.e., $f$ is subsmooth. Theorem A finishes the proof. 
Remarks. 1. It is easily seen that the above class of functions contains the class of lower $C^{1}$ functions. Combining this with Theorem 18 and Spingarn's characterization of lower $C^{1}$ functions ([24, Theorem 3.9]), we conclude that in finite dimensions a function $f$ is lower $C^{1}$ if, and only if, $f$ is given by (34) and satisfies conditions (i)-(iii).

2. If, in addition to the assumptions of Theorem [18, the choice of $\delta$ in (35) does not depend on $e$, then by [11, Theorem 5.4 (b)] we conclude that $\partial^{C} f$ is s-submonotone, and by Theorem $\mathrm{C}$, that $\partial^{F} f=\partial^{C} f$.

Now let $A$ be an arbitrary nonempty subset of the Banach space $X$. Let us define the distance function by

$$
d_{A}(x):=\inf _{\alpha \in A}\|x-\alpha\| \quad(x \in X) .
$$

The following proposition provides another typical example of subsmooth functions.

Proposition 19. Suppose that the norm of $X$ is uniformly Gâteaux (resp. uniformly Fréchet) differentiable. For any nonempty closed set $A$ of $X$, let us consider the function

$$
f(x)=-d_{A}(x)
$$

(i) Then $f$ is subsmooth, hence regular, on $X \backslash A$.

(ii) $\partial^{C} f=\partial^{H} f$ (resp. $\partial^{C} f=\partial^{F} f$ ) is maximal cyclically submonotone and $\partial^{C} d_{A}($.$) is minimal w^{*}$-cusco on $X \backslash A$.

Proof. The assertions follow from [11, Theorem 5.6 (a),(b)], Theorem A and Remark 2.

Let us now consider another important class of examples of subsmooth functions. We shall say that a function $f: U \rightarrow \mathbb{R}$ is amenable ([22, Definition 10.23]) if for any $x_{0} \in U$, there exist an open neighborhood $V$ of $x_{0}$, a Banach space $Y$, a continuously differentiable function $F: V \rightarrow Y$ and a proper lower semicontinuous convex function $g: Y \rightarrow \mathbb{R} \cup\{+\infty\}$ such that

$$
f(x)=g(F(x)) \text { for all } x \in V
$$

and

$$
\mathbb{R}_{+}\left(\operatorname{dom} g-F\left(x_{0}\right)\right)+F^{\prime}\left(x_{0}\right)(X)=Y .
$$

For the sake of simplicity, and since in this paper we limit our study to locally Lipschitz functions, we further consider the subclass $\mathcal{A}(U)$ of amenable functions $f$ such that in the decomposition (36) we have $F(V) \subseteq$ int $\operatorname{dom}(g)$. Obviously, every function in $\mathcal{A}(U)$ is locally Lipschitz and condition (37) is satisfied.

Proposition 20. If $f \in \mathcal{A}(U)$, then $\partial^{C} f$ is s-submonotone (hence, in particular, $f$ is subsmooth).

Proof. Let $f$ be in $\mathcal{A}(U)$. With no loss of generality, we may assume that $V=U$, so that $f=g \circ F$ with $g$ and $F$ as in (36). Since $g$ is regular on $F(U)$ (because it is convex and continuous on int $\operatorname{dom}(g)$ ), applying [6, Theorem 2.3.10] we conclude that $f$ is also regular, that is, $\partial^{C} f=\partial^{H} f$. Now set $T=\partial^{C} f=\partial^{H} f$. We shall show that $T$ is s-submonotone. 
To this end, let $x_{0} \in U, x_{1}, x_{2} \in U$ and $x_{i}^{*} \in \partial f\left(x_{i}\right), i=1,2$. Then there exist $y_{i}^{*} \in \partial g\left(F\left(x_{i}\right)\right)(i=1,2)$ such that $x_{i}^{*}=y_{i}^{*} \circ F^{\prime}\left(x_{i}\right)$, where $F^{\prime}(x)$ denotes the Fréchet derivative of $F$ at $x$. It follows that

$$
\left\langle x_{1}^{*}-x_{2}^{*}, x_{1}-x_{2}\right\rangle=\left\langle y_{1}^{*}, F^{\prime}\left(x_{1}\right)\left(x_{1}-x_{2}\right)\right\rangle-\left\langle y_{2}^{*}, F^{\prime}\left(x_{2}\right)\left(x_{1}-x_{2}\right)\right\rangle .
$$

Since $F$ is continuously differentiable, there exist $\delta>0$ and a function $r: U \times U \rightarrow Y$ such that

$$
F(v)-F(u)=F^{\prime}(u)(v-u)+r(u, v)
$$

for all $u, v \in B_{\delta}\left(x_{0}\right)$, and

$$
\lim _{\substack{u, v \rightarrow x_{0} \\ u \neq v}} \frac{\|r(u, v)\|}{\|u-v\|}=0 .
$$

Combining (38) with (39), thanks to the monotonicity of $\partial g$ we obtain

$$
\left\langle x_{1}^{*}-x_{2}^{*}, x_{1}-x_{2}\right\rangle \geq\left\langle y_{1}^{*}, r\left(x_{1}, x_{2}\right)\right\rangle+\left\langle y_{2}^{*}, r\left(x_{2}, x_{1}\right)\right\rangle,
$$

which yields, when $x_{1} \neq x_{2}$,

$$
\frac{\left\langle x_{1}^{*}-x_{2}^{*}, x_{1}-x_{2}\right\rangle}{\left\|x_{1}-x_{2}\right\|} \geq\left\langle y_{1}^{*}, \frac{r\left(x_{1}, x_{2}\right)}{\left\|x_{1}-x_{2}\right\|}\right\rangle+\left\langle y_{2}^{*}, \frac{r\left(x_{2}, x_{1}\right)}{\left\|x_{1}-x_{2}\right\|}\right\rangle .
$$

The result now follows from (40) and the local boundedness of $\partial g$ near $F\left(x_{0}\right)$.

Remark. Since every strictly Gâteaux differentiable function $F: U \rightarrow Y$ is locally Lipschitz (6. Proposition 2.2.1]), a slight modification of the above proof suffices to establish that $\partial^{C} f$ is ds-submonotone on $U$, whenever $F$ is strictly Gâteaux differentiable and $g$ is locally Lipschitz with $\partial^{C} g$ s-submonotone on an open set containing $F(U)$.

\section{REFERENCES}

1. Birge, R. And QI, L., Semi-regularity and generalized subdifferentials with applications to optimization, Math. Oper. Res. 18 (1993), 982-1005. MR 94h:49026

2. Borwein, J. M., Minimal cuscos and subgradients of Lipschitz functions, in: Fixed Point Theory and its Applications, (J.-B. Baillon and M. Théra, eds.), Pitman Res. Notes in Math. Series, No. 252, Longman, Essex, (1991), 57-82. MR 92j:46077

3. Borwein, J. And Moors, W., Essentially smooth Lipschitz functions, J. Funct. Anal. 149 (1997), 305-351. MR 98i:58028

4. Borwein, J., Moors, W. And Shao, Y., Subgradient representation of multifunctions, J. Austral. Math. Soc. (Series B) 40 (1998), 301-313. MR 2001b:49020

5. Borwein, J. AND ZHU, Q., Multivalued and functional analytic techniques in nonsmooth analysis, (F. H. Clarke and R. J. Stern, eds.), Nonlinear Analysis, Differential Equations and Control (1999), 61-157. MR 2002a:49016

6. Clarke, F. H., Optimization and Nonsmooth Analysis, Wiley Interscience, New York (1983). MR 85m:49002

7. Correa, R. And Jofre, A., Tangentially continuous directional derivatives in nonsmooth analysis, J. Opt. Th. Appl. 61 (1989), 1-21. MR 90h:49009

8. Correa, R. And Thibault, L., Subdifferential analysis of bivariate separately regular functions, J. Math. Anal. Appl. 148 (1990), 157-174. MR 91b:49018

9. Danillidis, A. And Hadjisavvas, N., On the subdifferentials of quasiconvex and pseudoconvex functions and cyclic monotonicity, J. Math. Anal. Appl. 237 (1999), 30-42. MR 2000h:49026

10. Georgiev, P., Submonotone mappings in Banach spaces and differentiability of nonconvex functions, Compt. Rend. Acad. Bulg. Sci. 42 (1989), 13-16. MR 90k:58013

11. Georgiev, P., Submonotone mappings in Banach spaces and applications, Set-Valued Analysis 5 (1997), 1-35. MR 98d:49021 
12. Janin, R., Sur des multiapplications qui sont des gradients généralisés, C.R. Acad. Sci. Paris 294 (1982), 117-119. MR 83d:58013

13. Lebourg, G., Generic differentiability of Lipschitzian functions, Trans. Amer. Math. Soc. 256 (1979), 125-144. MR 80i:58012

14. Mifflin, R., Semismooth and semiconvex functions in constrained optimization, SIAM J. Control Optim. 15 (1977), 959-972. MR 57:1541]

15. Moors, W., A characterization of minimal subdifferential mappings of locally Lipschitz functions, Set-Valued Analysis 3 (1995), 129-141. MR 96e:58013

16. Penot, J.-P., Favorable classes of mappings and multimappings in nonlinear analysis and optimization, J. Convex Analysis 3 (1996), 97-116. MR 97i:90110

17. Poliquin, R., Integration of subdifferentials of nonconvex functions, Nonlinear Analysis TMA 17 (1991), 385-398. MR 92i:49008

18. PreIss, D., Differentiability of Lipschitz functions on Banach spaces, J. Functional Analysis 91 (1990), 312-345. MR 91g:46051

19. QI, L., The maximal normal operator space and integration of subdifferentials of nonconvex functions, Nonlinear Analysis TMA 13 (1989), 1003-1011. MR 91a:90150

20. Rockafellar, R. T., On the maximal monotonicity of subdifferential mappings, Pacific J. Math. 33 (1970), 209-216. MR 41:7432

21. Rockafellar, R. T., "Favorable classes of Lipschitz continuous functions in subgradient optimization" in Nondifferentiable Optimization (1982), Nurminski E. (ed.), Pergamon Press, New York. MR 85e:90069

22. Rockafellar, R. T. And Wets, J.-B., Variational Analysis, Springer, New York (1998). MR 98m:49001

23. Preiss, D., Phelps, R. And Namioka, I., Smooth Banach spaces, weak Asplund spaces and monotone or USCO mappings, Israel J. Math. 72 (1990), 257-279. MR 92h:46021

24. Spingarn, J. E., Submonotone subdifferentials of Lipschitz functions, Trans. Amer. Math. Soc. 264 (1981), 77-89. MR 82g:26016

25. Thibault, L. and Zagrodny, D., Integration of subdifferentials of lower semi-continuous functions on Banach spaces, J. Math. Anal. Appl. 189 (1995), 33-58. MR 95i:49032

26. WANG, X., Fine and pathological properties of subdifferentials, Ph.D. Dissertation (1999), Simon Fraser University, Vancouver, Canada.

27. WU, Z. AND Ye, J., Some results on integration of subdifferentials, Nonlinear Analysis TMA 39 (2000), 955-976. MR 2000k:49022

Laboratoire de Mathématiques Appliquées, CNRS ERS 2055, Université de Pau et des Pays de l'Adour, aVenue de l'Université, 64000 Pau, France

E-mail address: aris.danillidis@univ-pau.fr

Current address: CODE - Edifici B, Universitat Autonoma de Barcelona, 08193 Bellaterra, Spain

Sofia University "St. Kl. Ohridski", Faculty of Mathematics and Informatics, 5 J. Bourchier Blvd., 1126 Sofia, Bulgaria

Current address: Laboratory for Advanced Brain Signal Processing, Brain Science Institute, The Institute of Physical and Chemical Research (RIKEN), 2-1, Hirosawa, Wako-shi, Saitama, 351-0198, Japan

E-mail address: georgiev@bsp.brain.riken.go.jp

Laboratoire de Mathématiques Appliquées, CNRS ERS 2055, Université de Pau et des

Pays de L'Adour, aVenue de l'Université, 64000 Pau, France

E-mail address: jean-paul.penot@univ-pau.fr 